\title{
On Invariant MASAs for Endomorphisms of the Cuntz Algebras
}

\author{
Jeong Hee Hong, Adam Skalski and Wojciech Szymański ${ }^{\dagger}$
}

10 January 2010

\begin{abstract}
The problem of existence of standard (i.e. product-type) invariant MASAs for endomorphisms of the Cuntz algebra $\mathcal{O}_{n}$ is studied. In particular endomorphisms which preserve the canonical diagonal MASA $\mathcal{D}_{n}$ are investigated. Conditions on a unitary $w \in \mathcal{U}\left(\mathcal{O}_{n}\right)$ equivalent to the fact that the corresponding endomorphism $\lambda_{w}$ preserves $\mathcal{D}_{n}$ are found, and it is shown that they may be satisfied by unitaries which do not normalize $\mathcal{D}_{n}$. Unitaries giving rise to endomorphisms which leave all standard MASAs invariant and have identical actions on them are characterized. Finally some properties of examples of finite-index endomorphisms of $\mathcal{O}_{n}$ given by Izumi and related to sector theory are discussed and it is shown that they lead to an endomorphism of $\mathcal{O}_{2}$ associated to a matrix unitary which does not preserve any standard MASA.
\end{abstract}

MSC 2010: 46L55, 46L40, 46L05

Keywords: Cuntz algebra, endomorphism, invariant MASA, diagonal.

*This work was supported by National Research Foundation of Korea Grant funded by the Korean Government (KRF-2008-313-C00039).

†This work was partially supported by the FNU Rammebevilling grant 'Operator algebras and applications' (2009-2011). 


\section{Introduction}

Systematic investigations of endomorphisms of the $C^{*}$-algebras $\mathcal{O}_{n}$ were initiated by Cuntz in [10], where he noticed the bijective correspondence between unitaries in $\mathcal{O}_{n}$ and unital endomorphisms of $\mathcal{O}_{n}$. From this, he derived a number of interesting properties of such endomorphisms.

Thirty years later, endomorphisms of $\mathcal{O}_{n}$ still constitute an active area of research offering a number of challenging problems and interesting connections with other fields, including index theory, noncommutative entropy, quantum groups and classical dynamical systems (see for example [3, 5, 11, 14, 19]). In particular, significant progress has been achieved recently in the study of endomorphisms corresponding to permutation unitaries, [4, 8, 17, 15, 16, 24, 25.

In [10], Cuntz considered also the following two problems: given a unitary $u \in \mathcal{O}_{n}$, under what conditions the corresponding endomorphism $\lambda_{u}$ globally preserves (i) the canonical UHF-subalgebra $\mathcal{F}_{n}$, and (ii) the canonical diagonal MASA $\mathcal{D}_{n}$ ? He gave the complete answers in the case when $\lambda_{u}$ is an automorphism of $\mathcal{O}_{n}$ and provided partial information in the general case when $\lambda_{u}$ is not necessarily surjective. The former problem has been recently successfully tackled in [6]. In the present note, we consider some issues closely related to the latter.

The main focus of the present paper are MASAs of $\mathcal{O}_{n}$ globally invariant under the action of a unital endomorphism. The best studied and most important endomorphism of $\mathcal{O}_{n}$, the canonical shift $\varphi$, is often viewed as the noncommutative generalization of the standard Bernoulli-Markov shift of the topological dynamics, as the restriction of $\varphi$ to the diagonal is indeed the map induced by the standard shift. As any standard MASA in $\mathcal{O}_{n}$ (i.e. the image of the canonical diagonal MASA $\mathcal{D}_{n}$ under a Bogolyubov automorphism) is naturally isomorphic to the algebra of continuous functions on the full shift on $n$-letters $\mathfrak{C}$, the usual arena of symbolic dynamics ([17]), it seems natural to ask what other classical transformations of $\mathfrak{C}$ one can obtain by restricting endomorphisms of $\mathcal{O}_{n}$ to invariant standard MASAs. It is easy to notice that the canonical shift leaves all standard MASAs invariant and always leads to the same transformation of $\mathfrak{C}$. The examples in [24] showed that there exist endomorphisms of $\mathcal{O}_{n}$ which reduce to the usual shift on $\mathfrak{C}$ in some invariant standard MASA, but lead to a different transformation in another. One could rephrase this in the following statement - a classical system arising in symbolic dynamics may have different 'quantizations' to 
endomorphisms of Cuntz algebras and at the same time an endomorphism of $\mathcal{O}_{n}$ may be a quantization of several different classical dynamical systems. A specific motivation for considering such questions comes from usefulness in the context of noncommutative entropy calculations of examining several different MASAs invariant under the same endomorphism (for example, see [24, 22]).

In this work we initiate a systematic study of the topics listed above. In particular, for a unital endomorphism $\rho$ of $\mathcal{O}_{n}$ we consider the question of how to identify all its $\rho$-invariant standard (product-type) MASAs. In Section 3 we show that this problem reduces to deciding which unitaries $w$ in $\mathcal{O}_{n}$ have the property that the corresponding endomorphism $\lambda_{w}$ globally preserves the diagonal $\mathcal{D}_{n}$. As already noted by Cuntz in [10], this is certainly the case when $w$ normalizes $\mathcal{D}_{n}$. In the main part of the present paper we consider this problem from a much more general perspective. In particular, our Theorem 4.1 gives a necessary and sufficient condition for a unitary $w$ in the algebraic part of $\mathcal{F}_{n}$ so that $\lambda_{w}\left(\mathcal{D}_{n}\right) \subseteq \mathcal{D}_{n}$. Verification of this condition involves only finitely many linear operations and could be easily performed by a computer. Corollaries to Theorem 4.1 offer further simplifications of the general conditions under additional assumptions and lead to a large class of examples of such endomorphisms corresponding to unitary matrices not belonging to the normalizer of $\mathcal{D}_{n}$.

In Section 5, we characterize the unitaries which are associated with the endomorphisms of $\mathcal{O}_{n}$ which leave all standard MASAs invariant and have identical actions on each of them. This is closely related to the work of Bratteli and Evans on the canonical $\mathcal{U}(n)$ action on $\mathcal{O}_{n}$ in [2], and earlier paper of Price on the action of $U(n)$ on the UHF algebra $\mathcal{F}_{n}$, [21. We note that commutation with Bogolyubov automorphisms has been exploited in the literature also in other contexts, see for example Section 4 in [14].

Section 6 is devoted to the analysis of examples of endomorphisms associated to finite abelian groups by Izumi in [14]. It turns out that Izumi's examples (arising from the subfactor theory), although not permutation endomorphisms themselves, are at the same time square roots of a permutation endomorphism and compositions of a permutation endomorphism with a Bogolyubov automorphism. In particular we obtain an endomorphism of $\mathcal{O}_{2}$ corresponding to a matrix unitary which does not globally preserve any standard MASA.

Acknowledgements. The first named author would like to thank Uffe 
Haagerup and other members of mathematics department at Odense for their warm hospitality during her stay there in the summer of 2009, when part of this work was done. The second named author's contribution to this note was made during his visit to University of Tokyo in October-November 2009, funded by a JSPS Short Term Postdoctoral Fellowship.

\section{Notation and preliminaries}

If $n$ is an integer greater than 1 , then the Cuntz algebra $\mathcal{O}_{n}$ is a unital, simple, purely infinite $C^{*}$-algebra generated by $n$ isometries $S_{1}, \ldots, S_{n}$, satisfying $\sum_{i=1}^{n} S_{i} S_{i}^{*}=I,[9]$. We denote by $W_{n}^{k}$ the set of $k$-tuples $\alpha=\left(\alpha_{1}, \ldots, \alpha_{k}\right)$ with $\alpha_{m} \in\{1, \ldots, n\}$, and by $W_{n}$ the union $\cup_{k=0}^{\infty} W_{n}^{k}$, where $W_{n}^{0}=\{0\}$. We call elements of $W_{n}$ multi-indices. If $\alpha \in W_{n}^{k}$ then $|\alpha|=k$ is the length of $\alpha$. If $\alpha=\left(\alpha_{1}, \ldots, \alpha_{k}\right) \in W_{n}$, then $S_{\alpha}=S_{\alpha_{1}} \ldots S_{\alpha_{k}}\left(S_{0}=I\right.$ by convention $)$ is an isometry with range projection $P_{\alpha}=S_{\alpha} S_{\alpha}^{*}$. Every word in $\left\{S_{i}, S_{i}^{*} \mid i=\right.$ $1, \ldots, n\}$ can be uniquely expressed as $S_{\alpha} S_{\beta}^{*}$, for $\alpha, \beta \in W_{n}$ [9, Lemma 1.3].

We denote by $\mathcal{F}_{n}^{k}$ the $C^{*}$-subalgebra of $\mathcal{O}_{n}$ spanned by all words of the form $S_{\alpha} S_{\beta}^{*}, \alpha, \beta \in W_{n}^{k}$, which is isomorphic to the matrix algebra $M_{n^{k}}(\mathbb{C})$. The norm closure $\mathcal{F}_{n}$ of $\cup_{k=0}^{\infty} \mathcal{F}_{n}^{k}$, is the UHF-algebra of type $n^{\infty}$, called the core UHF-subalgebra of $\mathcal{O}_{n}$, 9]. It is the fixed point algebra for the gauge action of the circle group $\gamma: U(1) \rightarrow \operatorname{Aut}\left(\mathcal{O}_{n}\right)$ defined on generators as $\gamma_{t}\left(S_{i}\right)=t S_{i}$. For $k \in \mathbb{Z}$, we denote by $\mathcal{O}_{n}^{(k)}:=\left\{x \in \mathcal{O}_{n}: \gamma_{t}(x)=t^{k} x\right\}$, the spectral subspace for this action. In particular, $\mathcal{F}_{n}=\mathcal{O}_{n}^{(0)}$. The $C^{*}$ subalgebra of $\mathcal{F}_{n}$ generated by projections $P_{\alpha}, \alpha \in W_{n}$, is a MASA (maximal abelian subalgebra) both in $\mathcal{F}_{n}$ and in $\mathcal{O}_{n}$. We call it the diagonal and denote $\mathcal{D}_{n}$. We also set $\mathcal{D}_{n}^{k}:=\mathcal{D}_{n} \cap \mathcal{F}_{n}^{k}$. Throughout this paper we are interested in the inclusions

$$
\mathcal{D}_{n} \subseteq \mathcal{F}_{n} \subseteq \mathcal{O}_{n}
$$

We denote by $\mathcal{S}_{n}$ the group of those unitaries in $\mathcal{O}_{n}$ which can be written as finite sums of words, i.e., in the form $u=\sum_{j=1}^{m} S_{\alpha_{j}} S_{\beta_{j}}^{*}$ for some $\alpha_{j}, \beta_{j} \in$ $W_{n}$. It turns out that $\mathcal{S}_{n}$ is isomorphic to the Higman-Thompson group $G_{n, 1}$ [1, 20]. We also denote $\mathcal{P}_{n}=\mathcal{S}_{n} \cap \mathcal{U}\left(\mathcal{F}_{n}\right)$. Then $\mathcal{P}_{n}=\cup_{k} \mathcal{P}_{n}^{k}$, where $\mathcal{P}_{n}^{k}$ are permutation unitaries in $\mathcal{U}\left(\mathcal{F}_{n}^{k}\right)$. That is, for each $u \in \mathcal{P}_{n}^{k}$ there is a unique permutation $\sigma$ of multi-indices $W_{n}^{k}$ such that

$$
u=\sum_{\alpha \in W_{n}^{k}} S_{\sigma(\alpha)} S_{\alpha}^{*} .
$$


As shown by Cuntz in [10], there exists the following bijective correspondence between unitaries in $\mathcal{O}_{n}$ and unital $*$-endomorphisms of $\mathcal{O}_{n}$ (whose collection we denote by $\left.\operatorname{End}\left(\mathcal{O}_{n}\right)\right)$. A unitary $u$ in $\mathcal{O}_{n}$ determines an endomorphism $\lambda_{u}$ by

$$
\lambda_{u}\left(S_{i}\right)=u S_{i}, \quad i=1, \ldots, n .
$$

Conversely, if $\rho: \mathcal{O}_{n} \rightarrow \mathcal{O}_{n}$ is an endomorphism, then $\sum_{i=1}^{n} \rho\left(S_{i}\right) S_{i}^{*}=u$ gives a unitary $u \in \mathcal{O}_{n}$ such that $\rho=\lambda_{u}$. If the unitary $u$ arises from a permutation $\sigma$ via the formula (1), the corresponding endomorphism will be sometimes denoted by $\lambda_{\sigma}$. Composition of endomorphisms corresponds to a 'convolution' multiplication of unitaries as follows:

$$
\lambda_{u} \circ \lambda_{w}=\lambda_{\lambda_{u}(w) u}
$$

We denote by $\varphi$ the canonical shift:

$$
\varphi(x)=\sum_{i} S_{i} x S_{i}^{*}, \quad x \in \mathcal{O}_{n} .
$$

If we take $u=\sum_{i, j} S_{i} S_{j} S_{i}^{*} S_{j}^{*}$ then $\varphi=\lambda_{u}$. It is well-known that $\varphi$ leaves invariant both $\mathcal{F}_{n}$ and $\mathcal{D}_{n}$, and that $\varphi$ commutes with the gauge action $\gamma$.

If $u \in \mathcal{U}\left(\mathcal{O}_{n}\right)$ then for each positive integer $k$ we denote

$$
u_{k}=u \varphi(u) \cdots \varphi^{k-1}(u) .
$$

We agree that $u_{k}^{*}$ stands for $\left(u_{k}\right)^{*}$. If $\alpha$ and $\beta$ are multi-indices of length $k$ and $m$, respectively, then $\lambda_{u}\left(S_{\alpha} S_{\beta}^{*}\right)=u_{k} S_{\alpha} S_{\beta}^{*} u_{m}^{*}$. This is established through a repeated application of the identity $S_{i} a=\varphi(a) S_{i}$, valid for all $i=1, \ldots, n$ and $a \in \mathcal{O}_{n}$.

Let $z \in \mathcal{U}\left(\mathcal{F}_{n}^{1}\right)$. Then $\lambda_{z}$ is an automorphism of $\mathcal{O}_{n}$ (with inverse $\lambda_{z^{*}}$ ), called a Bogolyubov automorphism. Since $z \in \mathcal{F}_{n}, \lambda_{z}$ restricts to an automorphism of $\mathcal{F}_{n}$. Each Bogolyubov automorphism acts as a unitary transformation on the Hilbert space $\operatorname{span}\left\{S_{i}: i=1, \ldots, n\right\}$ generating $\mathcal{O}_{n}$.

For algebras $A \subseteq B$ we denote by $\mathcal{N}_{B}(A)=\left\{u \in \mathcal{U}(B): u A u^{*}=A\right\}$ the normalizer of $A$ in $B$ and by $A^{\prime} \cap B=\left\{a \in A: \forall_{b \in B} a b=b a\right\}$ the relative commutant of $A$ in $B$.

\section{Standard invariant MASAs}

Let $z \in \mathcal{U}\left(\mathcal{F}_{n}^{1}\right)$. Then $\lambda_{z}$ is a Bogolyubov automorphism of $\mathcal{O}_{n}$ and $\mathcal{A}:=$ $\lambda_{z}\left(\mathcal{D}_{n}\right)$ is a MASA in $\mathcal{O}_{n}$ (and in $\left.\mathcal{F}_{n}\right)$. We will refer to MASAs of this 
form as standard. Every standard MASA is isomorphic to the $C^{*}$-algebra of continuous, complex-valued functions on the full shift on $n$ letters, denoted further by $\mathfrak{C}$ (as it is homeomorphic to a Cantor set).

Lemma 3.1. Let $z \in \mathcal{U}\left(\mathcal{F}_{n}^{1}\right)$ and denote $\mathcal{A}_{1}:=\lambda_{z}\left(\mathcal{D}_{n}^{1}\right)$. Then for each positive integer $k$ we have

$$
\lambda_{z}\left(\mathcal{D}_{n}^{k}\right)=\mathcal{A}_{1} \varphi\left(\mathcal{A}_{1}\right) \cdots \varphi^{k-1}\left(\mathcal{A}_{1}\right)
$$

Thus $\mathcal{A}$ is the increasing limit of algebras $\mathcal{A}_{1} \varphi\left(\mathcal{A}_{1}\right) \cdots \varphi^{k-1}\left(\mathcal{A}_{1}\right)$.

Proof. At first we verify by induction on $k$ that $\lambda_{z} \varphi^{k}\left(\mathcal{D}_{n}^{1}\right)=\varphi^{k}\left(\mathcal{A}_{1}\right)$. Indeed, for $k=1$ we have

$$
\lambda_{z} \varphi\left(\mathcal{D}_{n}^{1}\right)=z_{2} \varphi\left(\mathcal{D}_{n}^{1}\right) z_{2}^{*}=z \varphi\left(\mathcal{A}_{1}\right) z^{*}=\varphi\left(\mathcal{A}_{1}\right),
$$

since $z$ commutes with $\varphi\left(\mathcal{A}_{1}\right)$. Now suppose $\lambda_{z} \varphi^{k}\left(\mathcal{D}_{n}^{1}\right)=\varphi^{k}\left(\mathcal{A}_{1}\right)$. Then

$$
\lambda_{z} \varphi^{k+1}\left(\mathcal{D}_{n}^{1}\right)=z_{k+2} \varphi^{k+1}\left(\mathcal{D}_{n}^{1}\right) z_{k+2}^{*}=z \varphi\left(z_{k+1} \varphi^{k}\left(\mathcal{D}_{n}^{1}\right) z_{k+1}^{*}\right) z^{*}=\varphi^{k+1}\left(\mathcal{A}_{1}\right),
$$

since $z$ commutes with the range of $\varphi$.

Now we prove the lemma, again proceeding by induction on $k$. Case $k=1$ is just our definition of $\mathcal{A}_{1}$. Suppose $\lambda_{z}\left(\mathcal{D}_{n}^{k}\right)=\mathcal{A}_{1} \varphi\left(\mathcal{A}_{1}\right) \cdots \varphi^{k-1}\left(\mathcal{A}_{1}\right)$. Then we have

$$
\begin{gathered}
\lambda_{z}\left(\mathcal{D}_{n}^{k+1}\right)=\lambda_{z}\left(\mathcal{D}_{n}^{k} \varphi^{k}\left(\mathcal{D}_{n}^{1}\right)\right)=\lambda_{z}\left(\mathcal{D}_{n}^{k}\right) \lambda_{z} \varphi^{k}\left(\mathcal{D}_{n}^{1}\right) \\
=\mathcal{A}_{1} \varphi\left(\mathcal{A}_{1}\right) \cdots \varphi^{k-1}\left(\mathcal{A}_{1}\right) \varphi^{k}\left(\mathcal{A}_{1}\right)
\end{gathered}
$$

by the preceding observation.

It follows from Lemma 3.1 that if $w, z \in \mathcal{U}\left(\mathcal{F}_{n}^{1}\right)$ then

$$
\lambda_{w}\left(\mathcal{D}_{n}\right)=\lambda_{z}\left(\mathcal{D}_{n}\right) \Leftrightarrow w^{*} z \in \mathcal{N}_{\mathcal{F}_{n}^{1}}\left(\mathcal{D}_{n}^{1}\right) .
$$

In particular, $\lambda_{z}\left(\mathcal{D}_{n}\right)=\mathcal{D}_{n}$ if and only if $z \in \mathcal{N}_{\mathcal{F}_{n}^{1}}\left(\mathcal{D}_{n}^{1}\right)=\mathcal{P}_{n}^{1} \mathcal{U}\left(\mathcal{D}_{n}^{1}\right)$.

Proposition 3.2. Let $u \in \mathcal{U}\left(\mathcal{O}_{n}\right), z \in \mathcal{U}\left(\mathcal{F}_{n}^{1}\right)$, and set $\mathcal{A}=\lambda_{z}\left(\mathcal{D}_{n}\right)$. Then the following hold.

(i) $\lambda_{u}(\mathcal{A}) \subseteq \mathcal{A}$ if and only if $\lambda_{\lambda_{z^{*}}(u)}\left(\mathcal{D}_{n}\right) \subseteq \mathcal{D}_{n}$. In particular, if $\lambda_{z^{*}}(u) \in$ $\mathcal{N}_{\mathcal{O}_{n}}\left(\mathcal{D}_{n}\right)$ then $\lambda_{u}(\mathcal{A}) \subseteq \mathcal{A}$.

(ii) $\operatorname{Ad} u(\mathcal{A}) \subseteq \mathcal{A}$ if and only if $\lambda_{z^{*}}(u) \in \mathcal{N}_{\mathcal{O}_{n}}\left(\mathcal{D}_{n}\right)$. 
Proof. (i) Inclusion $\lambda_{u}(\mathcal{A}) \subseteq \mathcal{A}$ is equivalent to $\lambda_{z}^{-1} \lambda_{u} \lambda_{z}\left(\mathcal{D}_{n}\right) \subseteq \mathcal{D}_{n}$. But for $i=1, \ldots, n$ we have

$$
\begin{aligned}
\lambda_{z}^{-1} \lambda_{u} \lambda_{z}\left(S_{i}\right) & =\lambda_{z^{*}} \lambda_{u}\left(z S_{i}\right)=\lambda_{z^{*}} \lambda_{u}(z) \lambda_{u}\left(S_{i}\right)=\lambda_{z^{*}}\left(u z u^{*} \cdot u S_{i}\right)=\lambda_{z^{*}}\left(u z S_{i}\right) \\
& =\lambda_{z^{*}}(u) \lambda_{z^{*}}\left(z S_{i}\right)=\lambda_{z^{*}}(u) \lambda_{z^{*}} \lambda_{z}\left(S_{i}\right)=\lambda_{z^{*}}(u) S_{i}
\end{aligned}
$$

and thus $\lambda_{z}^{-1} \lambda_{u} \lambda_{z}=\lambda_{\lambda_{z^{*}}(u)}$. As shown in [10], if $\lambda_{z^{*}}(u)$ belongs to the normalizer of $\mathcal{D}_{n}$ then the endomorphism $\lambda_{\lambda_{z^{*}}(u)}$ globally preserves $\mathcal{D}_{n}$.

(ii) We have $\lambda_{z}^{-1} \operatorname{Ad} u \lambda_{z}=\operatorname{Ad} \lambda_{z^{*}}(u)$.

Consider a simple case of $u \in \mathcal{U}\left(\mathcal{F}_{n}^{1}\right)$. Then $\lambda_{z^{*}}(u)=z^{*} u z$ normalizes $\mathcal{D}_{n}$ if and only if $z^{*} u z$ is in $\mathcal{P}_{n}^{1} \mathcal{U}\left(\mathcal{D}_{n}^{1}\right)$. That is, in order to find MASAs of the form $\lambda_{z}\left(\mathcal{D}_{n}\right)$ which are globally invariant for $\lambda_{u}$ we would need to solve the generalized diagonalization problem for $u$ : find unitaries $z \in \mathcal{U}\left(\mathcal{F}_{n}^{1}\right)$ such that

$$
z^{*} u z=s d
$$

where $s \in \mathcal{P}_{n}^{1}$ is a permutation and $d \in \mathcal{U}\left(\mathcal{D}_{n}^{1}\right)$ is diagonal. More generally, if $u \in \mathcal{U}\left(\mathcal{F}_{n}^{k}\right)$, then we would be looking to solve the equation

$$
z_{k}^{*} u z_{k}=s d
$$

where $s \in \mathcal{P}_{n}^{k}, d \in \mathcal{U}\left(\mathcal{D}_{n}^{k}\right)$, and $z_{k}$ is a unitary defined for $z$ in formula (3)

The following example is a rephrasing in this language of the construction on pages $127-128$ in [24].

Example 3.3. Consider

$$
u=S_{1}\left(S_{1} S_{2}^{*}+S_{2} S_{1}^{*}\right) S_{1}^{*}+P_{2},
$$

a permutation unitary in $\mathcal{U}\left(\mathcal{F}_{2}^{2}\right)$. In particular, the diagonal $\mathcal{D}_{2}$ is invariant under $\lambda_{u}$. Take

$$
z=(1 / \sqrt{2})\left(1-S_{1} S_{2}^{*}+S_{2} S_{1}^{*}\right),
$$

a unitary in $\mathcal{U}\left(\mathcal{F}_{2}^{1}\right)$. Then one checks that

$$
\lambda_{z^{*}}(u)=z_{2}^{*} u z_{2}=S_{1} S_{1} S_{1}^{*} S_{1}^{*}+S_{1} S_{2} S_{2}^{*} S_{2}^{*}+S_{2} S_{2} S_{2}^{*} S_{1}^{*}+S_{2} S_{1} S_{1}^{*} S_{2}^{*}
$$

is a permutation in $\mathcal{P}_{2}^{2}$, and thus $\mathcal{A}=\lambda_{z}\left(\mathcal{D}_{2}\right)$ is an invariant MASA for $\lambda_{u}$. This MASA is different from the diagonal $\mathcal{D}_{2}$, since $z$ does not normalize $\mathcal{D}_{2}^{1}$. The endomorphism $\lambda_{u}$ does not preserve all standard masas: one can check that if $a \in[0,1], b=\sqrt{1-a^{2}}$, so that $z_{a}=a S_{1} S_{1}^{*}-b S_{1} S_{2}^{*}+b S_{2} S_{1}^{*}+a S_{2} S_{2}^{*}$ is a unitary in $\mathcal{F}_{2}^{1}$, then $\lambda_{u}$ preserves $\lambda_{z_{a}}\left(\mathcal{D}_{2}\right)$ if and only if $a \in\left\{0,1, \frac{1}{2} \sqrt{2}\right\}$. 
Remark 3.4. Let $u \in \mathcal{N}_{\mathcal{F}_{n}^{k}}\left(\mathcal{D}_{n}^{k}\right)$ and denote $C(u)=\left\{w \in \mathcal{U}\left(\mathcal{F}_{n}^{k}\right): w u=\right.$ $u w\}$, the centralizer of $u$ in $\mathcal{U}\left(\mathcal{F}_{n}^{k}\right)$. To find invariant MASAs for $\lambda_{u}$ one could look for unitaries $z \in \mathcal{U}\left(\mathcal{F}_{n}^{1}\right)$ such that $z_{k} \in C(u) \mathcal{N}_{\mathcal{F}_{n}^{k}}\left(\mathcal{D}_{n}^{k}\right)$. If such a $z$ does not normalize $\mathcal{D}_{n}^{1}$ then $\mathcal{A}=\lambda_{z}\left(\mathcal{D}_{n}\right)$ is different from $\mathcal{D}_{n}$.

Example 3.5. Consider the unitary

$$
u=S_{1} S_{1} S_{1}^{*}+S_{1} S_{2} S_{1}^{*} S_{2}^{*}+S_{2} S_{2}^{*} S_{2}^{*}
$$

in $\mathcal{S}_{2}$. The unitary $u$ is in the normalizer of the diagonal, i.e. $\operatorname{Ad} u\left(\mathcal{D}_{2}\right)=\mathcal{D}_{2}$. We ask if there exist other standard MASAs in $\mathcal{O}_{2}$ invariant under Adu. So let $\theta \in[0,2 \pi), a, b \in \mathbb{C}$ with $|a|^{2}+|b|^{2}=1$, and consider the unitary

$$
z=e^{i \theta} a S_{1} S_{1}^{*}+e^{i \theta} b S_{2} S_{1}^{*}-\bar{b} S_{1} S_{2}^{*}+\bar{a} S_{2} S_{2}^{*}
$$

in $\mathcal{U}\left(\mathcal{F}_{2}^{1}\right)$. According to Proposition 3.2, we must determine for what $z$ the unitary $\lambda_{z^{*}}(u)$ belongs to the normalizer of $\mathcal{D}_{2}$. In particular, for each projection $p \in \mathcal{D}_{2}$ we must have $\lambda_{z^{*}}(u) p \lambda_{z^{*}}(u)^{*} \in \mathcal{D}_{2}$. Decomposing $\lambda_{z^{*}}(u) p \lambda_{z^{*}}(u)^{*}$ as a sum of terms belonging to spectral subspaces $\mathcal{O}_{2}^{(k)}, k \in \mathbb{Z}$, we get that the term in $\mathcal{O}_{2}^{(2)}$ is $\lambda_{z^{*}}\left(S_{1} S_{1} S_{1}^{*}\right) p \lambda_{z^{*}}\left(S_{2} S_{2} S_{2}^{*}\right)$. Writing this expression explicitly for $p=S_{1} S_{1} S_{1}^{*} S_{1}^{*}$ (for example) we see that it is equal to 0 if and only if either $a=0$ or $b=0$. But then $z$ normalizes $\mathcal{D}_{2}$ and $\lambda_{z}\left(\mathcal{D}_{2}\right)=\mathcal{D}_{2}$. Therefore the only standard MASA of $\mathcal{O}_{2}$ invariant under $\operatorname{Ad} u$ is the diagonal $\mathcal{D}_{2}$.

As it turns out, there exist unitary elements of $\mathcal{F}_{n}^{k}$ whose associated endomorphisms do not leave invariant any standard MASA of $\mathcal{O}_{n}$. In Section 6. below, we show that this is the case for a certain endomorphism of $\mathcal{O}_{2}$ constructed by Izumi ([14]) in connection with Longo's sector theory ([18]) and Watatani's index theory for $C^{*}$-algebras ([26]).

\section{Endomorphisms preserving the diagonal}

Condition (i) of Proposition 3.2 motivates the following question: for what unitaries $w \in \mathcal{U}\left(\mathcal{O}_{n}\right)$ we have $\lambda_{w}\left(\mathcal{D}_{n}\right) \subseteq \mathcal{D}_{n}$ ? Certainly this is the case for those unitaries which normalize $\mathcal{D}_{n}$. An interesting question is what other unitaries, not belonging to $\mathcal{N}_{\mathcal{O}_{n}}\left(\mathcal{D}_{n}\right)$, give rise to endomorphisms which preserve the diagonal? Clearly, $\lambda_{w}\left(\mathcal{D}_{n}\right) \subseteq \mathcal{D}_{n}$ if and only if $\lambda_{w}\left(\mathcal{D}_{n}^{1}\right) \subseteq \mathcal{D}_{n}$ and $\lambda_{w} \varphi^{r}\left(\mathcal{D}_{n}^{1}\right) \subseteq \mathcal{D}_{n}$ for all $r=1,2, \ldots$. Unfortunately, these conditions are not easy to check in general. However, if $w$ is a unitary in the algebraic part 
of $\mathcal{F}_{n}$ then they reduce to a finite calculation, as explained in the following theorem.

Theorem 4.1. Let $k \in \mathbb{N}$ and let $w \in \mathcal{U}\left(\mathcal{F}_{n}^{k}\right)$. For $i, j=1, \ldots, n$ let $E_{i j}: \mathcal{F}_{n}^{k} \rightarrow \mathcal{F}_{n}^{k-1}$ be linear maps determined by the condition that $a=$ $\sum_{i, j=1}^{n} E_{i j}(a) \varphi^{k-1}\left(S_{i} S_{j}^{*}\right)$ for all $a \in \mathcal{F}_{n}^{k}$. Define by induction an increasing sequence of unital selfadjoint subspaces $\mathfrak{S}_{r}$ of $\mathcal{F}_{n}^{k-1}$ so that

$$
\begin{aligned}
\mathfrak{S}_{1} & =\operatorname{span}\left\{E_{j j}\left(w x w^{*}\right): x \in \mathcal{D}_{n}^{1}, j=1, \ldots, n\right\}, \\
\widetilde{\mathfrak{S}}_{r+1} & =\operatorname{span}\left\{E_{j j}((\operatorname{Ad} w \circ \varphi)(x)): x \in \mathfrak{S}_{r}, j=1, \ldots, n\right\}, \\
\mathfrak{S}_{r+1} & =\mathfrak{S}_{r}+\widetilde{\mathfrak{S}}_{r+1} .
\end{aligned}
$$

We agree that $\mathfrak{S}_{0}=\mathbb{C} 1$. Let $R$ be the smallest integer such that $\mathfrak{S}_{R}=\mathfrak{S}_{R-1}$. Then $\lambda_{w}\left(\mathcal{D}_{n}\right) \subseteq \mathcal{D}_{n}$ if and only if $\lambda_{w}\left(\mathcal{D}_{n}^{R}\right) \subseteq \mathcal{D}_{n}$.

Proof. Let $\lambda_{w}\left(\mathcal{D}_{n}^{R}\right) \subseteq \mathcal{D}_{n}$. We show by induction on $r=1,2, \ldots$ that

(i) $\mathfrak{S}_{r} \subseteq \mathcal{D}_{n}^{k-1}$

(ii) $(\operatorname{Ad} w \circ \varphi)\left(\mathfrak{S}_{r-1}\right) \subseteq \mathfrak{S}_{r} \varphi^{k-1}\left(\mathcal{D}_{n}^{1}\right)$;

(iii) $\lambda_{w} \varphi^{r-1}\left(\mathcal{D}_{n}^{1}\right) \subseteq \mathcal{D}_{n}$;

(iv) for each $x \in \mathcal{D}_{n}^{1}$ and $r$ there exist elements $x_{j_{1} \ldots j_{r}}$ of $\mathfrak{S}_{r}$ such that

$$
\lambda_{w} \varphi^{r-1}(x)=\sum_{j_{1}, \ldots, j_{r}=1}^{n} x_{j_{1} \ldots j_{r}} \varphi^{k-1}\left(P_{j_{r}}\right) \varphi^{k}\left(P_{j_{r-1}}\right) \cdots \varphi^{k+r-2}\left(P_{j_{1}}\right)
$$

(v) $\mathfrak{S}_{r}$ is spanned by $\mathfrak{S}_{r-1}$ and elements $x_{j_{1} \ldots j_{r}}$ appearing in formula (4) for all $x \in \mathcal{D}_{n}^{1}$.

Base step $r=1$. By hypothesis, $\lambda_{w}\left(\mathcal{D}_{n}^{1}\right)=w \mathcal{D}_{n}^{1} w^{*}$ is contained in $\mathcal{D}_{n}^{k}$. Thus for $x \in \mathcal{D}_{n}^{1}$ we have

$$
\lambda_{w}(x)=\sum_{j=1}^{n} E_{j j}\left(w x w^{*}\right) \varphi^{k-1}\left(P_{j}\right)
$$

and all $E_{j j}\left(w x w^{*}\right)$ must belong to $\mathcal{D}_{n}^{k-1}$. Whence $\mathfrak{S}_{1} \subseteq \mathcal{D}_{n}^{k-1}$ and formula (4) holds for $r=1$. Condition (v) follows. 
For the inductive step, suppose the claim holds for all $m=1, \ldots, r$. Let $x \in \mathcal{D}_{n}^{1}$. Then by the inductive hypothesis, and taking into account that $w$ commutes with the range of $\varphi^{l}$ if $l \geq k$, we get

$$
\begin{aligned}
\lambda_{w} \varphi^{r}(x) & =(\operatorname{Ad} w \circ \varphi) \lambda_{w} \varphi^{r-1}(x) \\
& =\sum_{j_{1}, \ldots, j_{r}=1}^{n}(\operatorname{Ad} w \circ \varphi)\left(x_{j_{1} \ldots j_{r}}\right) \varphi^{k}\left(P_{j_{r}}\right) \cdots \varphi^{k+r-1}\left(P_{j_{1}}\right)
\end{aligned}
$$

for some $x_{j_{1} \ldots j_{r}} \in \mathfrak{S}_{r}$. Firstly, suppose that $r \leq R-1$. Then $\varphi^{r}(x) \in \mathcal{D}_{n}^{R}$ and $\lambda_{w} \varphi^{r}(x)$ belongs to $\mathcal{D}_{n}$, by assumption. Thus each $(\operatorname{Ad} w \circ \varphi)\left(x_{j_{1} \ldots j_{r}}\right)$ is in $\mathcal{D}_{n}^{k}$ and we have

$$
(\operatorname{Ad} w \circ \varphi)\left(x_{j_{1} \ldots j_{r}}\right)=\sum_{j=1}^{n} E_{j j}\left((\operatorname{Ad} w \circ \varphi)\left(x_{j_{1} \ldots j_{r}}\right)\right) \varphi^{k-1}\left(P_{j}\right)
$$

Setting

$$
x_{j_{1} \ldots j_{r} j_{r+1}}=E_{j_{r+1} j_{r+1}}\left((\operatorname{Ad} w \circ \varphi)\left(x_{j_{1} \ldots j_{r}}\right)\right)
$$

we see that formula (4) holds for $r+1$, and also that $\mathfrak{S}_{r+1} \subseteq \mathcal{D}_{n}^{k-1}$ and $(\operatorname{Ad} w \circ \varphi)\left(\mathfrak{S}_{r}\right) \subseteq \mathfrak{S}_{r+1} \varphi^{k-1}\left(\mathcal{D}_{n}^{1}\right)$. This establishes the inductive step in the present case.

Secondly, suppose that $r \geq R$. Then all elements $x_{j_{1} \ldots j_{r}}$ belong to $\mathfrak{S}_{r}=\mathfrak{S}_{r-1}$. Thus, by the inductive hypothesis, $(\operatorname{Ad} w \circ \varphi)\left(x_{j_{1} \ldots j_{r}}\right)$ is in $\mathfrak{S}_{r} \varphi^{k-1}\left(\mathcal{D}_{n}^{1}\right) \subseteq \mathcal{D}_{n}^{k-1} \varphi^{k-1}\left(\mathcal{D}_{n}^{1}\right)$. Consequently, $\lambda_{w} \varphi^{r}(x)$ belongs to $\mathcal{D}_{n}$, and the rest of the argument is similar to the preceding case.

Validity of condition (iii) for all $r \in \mathbb{N}$ implies that $\lambda_{w}\left(\mathcal{D}_{n}\right) \subset \mathcal{D}_{n}$ and the proof is finished.

The simplest possible case to consider from the point of view of Theorem 4.1 is when already $\mathfrak{S}_{1}=\mathfrak{S}_{0}$. This leads to the following.

Corollary 4.2. Let $w$ be a unitary element of $\mathcal{F}_{n}^{k}$. If $w \mathcal{D}_{n}^{1} w^{*}=\varphi^{k-1}\left(\mathcal{D}_{n}^{1}\right)$ then $\lambda_{w}\left(\mathcal{D}_{n}\right) \subseteq \mathcal{D}_{n}$.

Proof. Indeed, if $w \mathcal{D}_{n}^{1} w^{*}=\varphi^{k-1}\left(\mathcal{D}_{n}^{1}\right)$ then $\mathfrak{S}_{1}=\mathfrak{S}_{0}$ and $\lambda_{w}\left(\mathcal{D}_{n}^{1}\right) \subseteq \mathcal{D}_{n}$.

Corollary 4.2 and Proposition 3.2 lead to the following explicit condition guaranteeing that the endomorphism associated to a unitary in the algebraic part of $\mathcal{F}_{n}$ leaves a given standard MASA invariant. 
Corollary 4.3. Let $u \in \mathcal{F}_{n}^{k}$ and $z \in \mathcal{U}\left(\mathcal{F}_{n}^{1}\right)$. If $u\left(z \mathcal{D}_{n}^{1} z^{*}\right) u^{*}=\varphi^{k-1}\left(z \mathcal{D}_{n}^{1} z^{*}\right)$ then $\mathcal{A}=\lambda_{z}\left(\mathcal{D}_{n}^{1}\right)$ is $\lambda_{u}$-invariant.

Example 4.4. One notices that Corollary 4.2 easily gives examples of unitaries $w$ such that $\lambda_{w}\left(\mathcal{D}_{n}\right) \subseteq \mathcal{D}_{n}$ but $w \notin \mathcal{N}_{\mathcal{O}_{n}}\left(\mathcal{D}_{n}\right)$. For instance, if $a, b, c, d \in \mathbb{C}$ are such that $|a|^{2}+|b|^{2}=|c|^{2}+|d|^{2}=1$ then

$$
\begin{aligned}
w & =S_{1}\left(a S_{1} S_{1}^{*}+b S_{1} S_{2}^{*}\right) S_{1}^{*}+S_{1}\left(c S_{2} S_{1}^{*}+d S_{2} S_{2}^{*}\right) S_{2}^{*} \\
& +S_{2}\left(-\bar{b} S_{1} S_{1}^{*}+\bar{a} S_{1} S_{2}^{*}\right) S_{1}^{*}+S_{2}\left(-\bar{d} S_{2} S_{1}^{*}+\bar{c} S_{2} S_{2}^{*}\right) S_{2}^{*}
\end{aligned}
$$

satisfies the condition of Corollary 4.2 (with $n=k=2$ ) and thus $\lambda_{w}\left(\mathcal{D}_{2}\right) \subseteq$ $\mathcal{D}_{2}$, but $w$ belongs to $\mathcal{N}_{\mathcal{O}_{2}}\left(\mathcal{D}_{2}\right)$ only if $a b=c d=0$.

Motivated by [24] (where a similar construction was used when an endomorphism different from the canonical shift was shown to reduce to the classical shift on a non-diagonal invariant standard MASA) we also consider the following sufficient condition which guarantees that $\lambda_{w}\left(\mathcal{D}_{n}\right) \subseteq \mathcal{D}_{n}$ :

$[\mathrm{D} 1] \lambda_{w}\left(\mathcal{D}_{n}^{1}\right) \subseteq \mathcal{D}_{n}$

[D2] $\lambda_{w} \varphi^{r}(x)=\varphi^{r} \lambda_{w}(x)$ for all $x \in \mathcal{D}_{n}^{1}$ and $r=1,2, \ldots$

A simple inductive argument shows that

$[\mathrm{D} 2] \Leftrightarrow w \in \bigcap_{r=1}^{\infty}\left(\varphi^{r} \lambda_{w}\left(\mathcal{D}_{n}^{1}\right)\right)^{\prime} \cap \mathcal{O}_{n}$.

Thus, since $\mathcal{F}_{n}^{k}$ and $\varphi^{k}\left(\mathcal{O}_{n}\right)$ commute, for $w \in \mathcal{U}\left(\mathcal{F}_{n}^{k}\right)$ conditions [D1] and [D2] are equivalent to the following:

$[\mathrm{DF} 1] w \mathcal{D}_{n}^{1} w^{*} \subseteq \mathcal{D}_{n}^{k}$

$[\mathrm{DF} 2] w \in \bigcap_{r=1}^{k-1}\left(\varphi^{r}\left(w \mathcal{D}_{n}^{1} w^{*}\right)\right)^{\prime} \cap \mathcal{F}_{n}^{k}$.

In this way we obtain the following.

Proposition 4.5. Let $w$ be a unitary element of $\mathcal{F}_{n}^{k}$. Denote by $B_{w}$ the $C^{*}$-algebra generated by $\left\{\varphi^{r}\left(w x w^{*}\right): x \in \mathcal{D}_{n}^{1}, r=1, \ldots, k-1\right\}$. If

$$
w \mathcal{D}_{n}^{1} w^{*} \subseteq \mathcal{D}_{n}^{k} \quad \text { and } \quad w \in B_{w}^{\prime} \cap \mathcal{F}_{n}^{k}
$$

then $\lambda_{w}\left(\mathcal{D}_{n}\right) \subseteq \mathcal{D}_{n}$

A very special case of the situation described in the preceding proposition takes place when $w \in \mathcal{U}\left(\mathcal{F}_{n}^{k}\right)$ and $w \mathcal{D}_{n}^{1} w^{*}=\varphi^{k-1}\left(\mathcal{D}_{n}^{1}\right)$, the case considered in Corollary 4.2. 


\section{Endomorphisms commuting with Bogolyubov automorphisms}

The methods of computing entropy of permutation endomorphisms of $O_{n}$ used in [24] were based on analysis of the behaviour of the transformations in question on invariant standard MASAs. It was noted there that even in simple cases entropy properties of $\left.\rho\right|_{\mathcal{A}_{1}}$ and $\left.\rho\right|_{\mathcal{A}_{2}}$ for two invariant standard MASAs $\mathcal{A}_{1}$ and $\mathcal{A}_{2}$ can be very different. In this section, we discuss endomorphisms of $\mathcal{O}_{n}$ whose restrictions to all standard MASAs are identical.

We begin with a simple and well-known observation:

Lemma 5.1. Let $u \in \mathcal{U}\left(\mathcal{O}_{n}\right)$. If the endomorphism $\lambda_{u}$ commutes with the gauge action, then $u \in \mathcal{F}_{n}$.

Proof. The assumed commutation relation implies that for each $i=1, \ldots, n$ and $t \in U(1)$ we have

$$
\gamma_{t}(u) \gamma_{t}\left(S_{i}\right)=u \gamma_{t}\left(S_{i}\right)
$$

This implies that $\gamma_{t}(u)=u$ for all $t \in U(1)$ and thus $u \in \mathcal{F}_{n}$.

By virtue of the above lemma, endomorphisms commuting with the gauge action leave the core UHF subalgebra $\mathcal{F}_{n}$ invariant. Interesting examples of endomorphisms of $\mathcal{O}_{n}$ that leave $\mathcal{F}_{n}$ invariant and yet their associated unitaries are not in $\mathcal{F}_{n}$ were recently found in [6].

We denote by $\operatorname{Sym}(k)$ the group of all permutations of $k$ letters.

Definition 5.2. Let $\sigma$ be a permutation of $W_{n}^{k}$. We call $\sigma$ induced if there exists a permutation $\omega \in \operatorname{Sym}(k)$ such that for all $\alpha \in W_{n}^{k}$ we have

$$
\sigma(\alpha)=\left(\alpha_{\omega(1)}, \ldots, \alpha_{\omega(k)}\right) .
$$

The following fact is a consequence of the classical theorem of Hermann Weyl.

Lemma 5.3. Let $k \in \mathbb{N}$ and let $u$ be a unitary in $\mathcal{F}_{n}^{k}$. The endomorphism $\lambda_{u}$ commutes with all Bogolyubov automorphisms of $\mathcal{O}_{n}$ if and only if $u$ is a linear combination of permutation unitaries associated with induced permutations of $W_{n}^{k}$. 
Proof. Let $u$ be as in the statement of the lemma and let $z \in \mathcal{U}\left(\mathcal{F}_{n}^{1}\right)$, so that $\lambda_{z}$ is a Bogolyubov automorphism of $\mathcal{O}_{n}$. By (2), endomorphisms $\lambda_{u}$ and $\lambda_{z}$ commute if and only if

$$
\lambda_{u}(z) u=\lambda_{z}(u) z .
$$

Since $\lambda_{u}(z)=u z u^{*}$ and $\lambda_{z}(u)=z_{k} u z_{k}^{*}$ (with $z_{k}$ as in (3)), this is equivalent to

$$
u=z_{k} u z_{k}^{*}
$$

Under the identification $\mathcal{F}_{n}^{k} \cong M_{n}(\mathbb{C})^{\otimes k}$, unitary $z_{k}$ corresponds to the tensor product $z^{\otimes k}$. Then equation (5) takes the form

$$
u=z^{\otimes k} u z^{* \otimes k} .
$$

View now this equation as an equation for a fixed matrix $u \in M_{n}(\mathbb{C})^{\otimes k}$ and arbitrary unitary $z \in M_{n}(\mathbb{C})$. It means that

$$
u \in\left\{z^{\otimes k}: z \in U(n)\right\}^{\prime}=\left\{z^{\otimes k}: z \in S U(n)\right\}^{\prime},
$$

so that $u$ is in the commutant of the $k$-th tensor power of the standard representation of $S U(n)$. A classical result of Weyl says that this commutant is equal to the image of the natural representation of the permutation group $\operatorname{Sym}(k)$ in $M_{n}(\mathbb{C})^{\otimes k}$ (see $\S 9.1 .1$ in $\left.[13]\right)$. This means that $u$ commutes with all the Bogolyubov automorphisms if and only if it is a linear combination of unitary matrices representating the permutations in $\operatorname{Sym}(k)$. It remains to observe that under the identification of $\mathcal{F}_{n}^{k}$ with $M_{n}(\mathbb{C})^{\otimes k}$ the latter matrices correspond to the unitaries associated with induced permutations.

Lemmas 5.1 and 5.3 almost characterize unitaries that lead to endomorphisms commuting with all Bogolyubov automorphisms; we can also have $u \in \mathcal{F}_{n} \backslash \bigcup_{k \in \mathbb{N}} \mathcal{F}_{n}^{k}$, but this can happen only in a trivial way (i.e. $u$ will be a norm limit of unitaries of the type described in Lemma 5.3). This has been observed (in the context of the UHF algebras) by Price in [21]. We formulate it in the next theorem; for the completeness we also present the proof. Let

$$
\mathbf{F}=\bigcap_{z \in \mathcal{U}\left(\mathcal{F}_{n}^{1}\right)}\left\{x \in \mathcal{O}_{n}: \lambda_{z}(x)=x\right\}
$$

Theorem 5.4. Let $u \in \mathcal{O}_{n}$ be unitary. The endomorphism $\lambda_{u}$ commutes with all Bogolyubov automorphisms of $\mathcal{O}_{n}$ if and only if $u$ is in the closed linear span of unitaries associated with induced permutations. 
Proof. By Lemmas 5.1 and 5.3 together with their proofs it suffices to show that $\mathbf{F} \cap \bigcup_{\mathbf{k} \in \mathbb{N}} \mathcal{F}_{\mathbf{n}}^{\mathbf{k}}$ is dense in $\mathbf{F}$. Let $\tau \in \mathcal{F}_{n}^{*}$ denote the faithful trace of the UHF algebra $\mathcal{F}_{n}$. Let $k \in \mathbb{N}$. With respect to the natural isomorphism $\iota_{k}$ : $\mathcal{F}_{n} \rightarrow \mathcal{F}_{n} \otimes M_{n}{ }^{\otimes k}$ we have $\tau=\left(\tau \otimes \operatorname{tr}_{n^{k}}\right) \circ \iota_{k}$, where $\operatorname{tr}_{n^{k}}$ is the normalised trace on $M_{n}{ }^{\otimes k}$. The identification of $M_{n}{ }^{\otimes k}$ with $\mathcal{F}_{n}^{k}$ in this picture corresponds to the mapping $\theta_{k}: M_{n}{ }^{\otimes k} \rightarrow \mathcal{F}_{n}$ defined by $\theta_{k}(x)=\iota_{k}{ }^{-1}\left(1_{\mathcal{F}_{n}} \otimes x\right), x \in M_{n}{ }^{\otimes k}$. Consider the conditional expectation $E_{k}: \mathcal{F}_{n} \rightarrow \mathcal{F}_{n}^{k}$ given by

$$
E_{k}=\theta_{k} \circ\left(\tau \otimes \mathrm{id}_{M_{n} \otimes k}\right) \circ \iota_{k} .
$$

As $\bigcup_{k \in \mathbb{N}} \mathcal{F}_{n}^{k}$ is dense in $\mathcal{F}_{n}$,

$$
E_{k}(x) \stackrel{k \rightarrow \infty}{\longrightarrow} x, \quad x \in \mathcal{F}_{n} .
$$

Let $\lambda_{z}$ be a Bogolyubov automorphism $\left(z \in \mathcal{F}_{n}^{1}\right)$. Then $E_{k} \circ \lambda_{z}=\lambda_{z} \circ E_{k}$ - the commutation relation is easily checked on elements in $\mathcal{F}_{n}^{l}(l \geq k)$ and by contractivity of the maps involved has to hold on the whole $\mathcal{F}_{n}$. This implies that for each $k \in \mathbb{N}$ there is $E_{k}(\mathbf{F})=\mathbf{F}$, which together with the displayed formula yields the statement formulated in the first sentence of the proof.

The above theorem, or rather its proof, shows that we have (recall the notation in (7))

$$
\mathbf{F} \cap \mathcal{O}_{\mathbf{n}}=\mathbf{F} \cap \mathcal{F}_{\mathbf{n}} \approx \mathbf{C}^{*}(\operatorname{Sym}(\infty))
$$

(with $\operatorname{Sym}(\infty)$ denoting the group of finite permutations of $\mathbb{N}$ ), as noted by Bratteli and Evans in [2].

Corollary 5.5. Let $\sigma$ be a permutation of $W_{n}^{k}$. Then the corresponding endomorphism $\lambda_{\sigma}$ commutes with all Bogolyubov automorphisms of $\mathcal{O}_{n}$ if and only if $\sigma$ is induced.

Proof. Follows from Lemma 5.3 and a simple observation that a permutation unitary in $\mathcal{F}_{n}^{k}$ can be a linear combination of permutation unitaries in $\mathcal{F}_{n}^{k}$ only in a trivial way.

Thus the class of induced permutation endomorphisms is the class of endomorphisms that not only leave each standard MASA invariant, but also induce the same homeomorphisms of the underlying Cantor set in each case. When $k=2$, induced permutation endomorphisms are the identity morphism 
and the canonical shift, but already when $k=3$ we get some new maps, for example the endomorphism associated to the unitary $\sum_{i, j, l=1}^{n} S_{l} S_{j} S_{i} S_{j}^{*} S_{i}^{*} S_{l}^{*}$. Note that the product of induced permutation endomorphisms is an induced permutation endomorphism, with the action on the level of permutations in $\bigcup_{k \in \mathbb{N}} \operatorname{Sym}(k)$ given by the convolution multiplication described by formula (7) of [25] (note that in [25] the convolution multiplication is defined for permutations of multi-indices). Each such endomorphism on the diagonal (or any other standard MASA) reduces to a transformation which has a simple combinatorial description as a substitution map.

Comment 5.6. In view of the discussion in [23] and the simple form of the induced permutation endomorphisms it is natural to expect that for such endomorphisms their Voiculescu topological entropy can be detected already from the restriction to the canonical diagonal MASA. We also conjecture that for an endomorphism $\lambda$ associated to an induced permutation the entropies of $\lambda$ and $\lambda \circ \alpha$ coincide for all Bogolyubov automorphisms $\alpha$. Note that in that situation we have $(\lambda \circ \alpha)^{m}=\lambda^{m} \circ \alpha^{m}$. A combination of results in Section 6. below, and a straightforward symbolic dynamics computation allowed to show in [22] that in general for an endomorphism $\lambda$ of $\mathcal{O}_{n}$ (even induced by a unitary in $\mathcal{F}_{n}$ ) and a Bogolyubov automorphism $\alpha$ the entropies of $\lambda$ and $\lambda \circ \alpha$ may be different (although, as follows from [12], the entropy of $\alpha$ is 0 ).

Finally, we observe that there exist endomorphisms preserving all standard MASAs which nevertheless do not commute with all Bogolyubov automorphisms, as the following example demonstrates.

Example 5.7. Let $k \geq 2$ and let $\sigma \in \operatorname{Sym}(k)$ be such that $\sigma(1)=k$ and $\sigma(k)=1$. Let $u_{1}$ be the unitary in $\mathcal{F}_{n}^{k}$ induced by $\sigma$, and let $u_{2}$ be an arbitray unitary in $\varphi\left(\mathcal{F}_{n}^{k-1}\right)$. Set $u=u_{1} u_{2}$. Then for any $z \in \mathcal{U}\left(\mathcal{F}_{n}^{1}\right)$ we have $\lambda_{z}\left(u_{1}\right)=u_{1}$ and $\lambda_{z}\left(u_{2}\right) \in \varphi\left(\mathcal{F}_{n}^{k-1}\right)$. Note that for each $i, j=1, \ldots, n$ there is $u_{1} S_{i} S_{j}^{*}=\varphi^{k-1}\left(S_{i} S_{j}^{*}\right) u_{1}$. Consequently,

$$
\lambda_{z}(u) \mathcal{D}_{n}^{1} \lambda_{z}\left(u^{*}\right)=u_{1} \lambda_{z}\left(u_{2}\right) \mathcal{D}_{n}^{1} \lambda_{z}\left(u_{2}^{*}\right) u_{1}^{*}=u_{1} \mathcal{D}_{n}^{1} u_{1}^{*}=\varphi^{k-1}\left(\mathcal{D}_{n}^{1}\right) .
$$

Thus $\lambda_{u}$ preserves all standard MASAs by Corollary 4.3. However, using freedom of choice of $u_{2}$, one can arrange that $u$ does not satisfy conditions of Lemma 5.3 (or Corollary 5.5), and thus it does not commute with all Bogolyubov automorphisms. A particular example of such situation is given by $(n=2) u_{1}=S_{1} S_{1} S_{1}^{*} S_{1}^{*}+S_{1} S_{2} S_{1}^{*} S_{2}^{*}+S_{2} S_{1} S_{2}^{*} S_{1}^{*}+S_{2} S_{2} S_{2} S_{2}^{*}$, 
$u_{2}=S_{1} S_{1} S_{1}^{*} S_{2}^{*}+S_{1} S_{2} S_{1}^{*} S_{1}^{*}+S_{2} S_{1} S_{2} S_{2}^{*}+S_{2} S_{2} S_{2}^{*} S_{1}^{*}$. The endomorphism $\lambda_{u_{1} u_{2}}$ is then a permutation endomorphism denoted by $\rho_{1342}$ in [24. We recommend comparing its properties with these of the endomorphism described in Example 3.3.

\section{Izumi's examples of real sectors}

In [14, Izumi studied certain explicit examples of endomorphisms of Cuntz algebras motivated by subfactor theory. His examples are square roots of canonical endomorphisms in the sense of Longo ([18]) with finite Watatani indices $([26])$. Izumi's methods are based on the following proposition, which is a special case of Proposition 2.5 in [14]:

Proposition 6.1 ([14]). Let $\lambda \in \operatorname{End}\left(\mathcal{O}_{n}\right)$. If there exists an isometry $w \in$ $\mathcal{O}_{n}$ such that $w x=\lambda^{2}(x) w$ for all $x \in \mathcal{O}_{n}$ and $w^{*} \lambda(w)=\frac{1}{d}$ for some $d>0$, then $E_{\lambda}: \mathcal{O}_{n} \rightarrow \lambda\left(\mathcal{O}_{n}\right)$ defined by

$$
E_{\lambda}(x)=\lambda\left(w^{*} \lambda(x) w\right), \quad x \in \mathcal{O}_{n},
$$

is a conditional expectation with Watatani index $d^{2}$.

Endomorphism $\lambda$ as above is called a real sector, as it is its own conjugate endomorphism ([18]). Examples of real sectors satisfying the conditions in the above proposition cannot be obtained for $w=S_{i}$ and $\lambda$ being a permutation endomorphism. Nevertheless, the endomorphism constructed in Example 3.7 of [14] is of the form $\lambda=\lambda_{\sigma} \circ \beta$, where $\beta$ is a Bogolyubov automorphism; moreover $\lambda^{2}$ is also a permutation endomorphism. Below we give proofs of these facts.

Let $G$ be a finite abelian group of cardinality $n$, written additively. Let $\langle\cdot, \cdot\rangle: G \times G \rightarrow \mathbb{T}$ be a symmetric duality bracket satisfying the usual conditions $\left(g, g^{\prime}, h \in G\right)$

$$
\begin{gathered}
\overline{\langle g, h\rangle}=\langle-g, h\rangle, \quad \sum_{h \in G}\langle h, g\rangle= \begin{cases}0 & \text { if } g \neq e \\
N & \text { if } g=e\end{cases} \\
\langle g, h\rangle\left\langle g^{\prime}, h\right\rangle=\left\langle g+g^{\prime}, h\right\rangle,\langle g, h\rangle=\langle h, g\rangle .
\end{gathered}
$$

If $G=\mathbb{Z}_{n}$ is a cyclic group then one can put $\langle k, l\rangle:=\exp \left(\frac{2 \pi i(k l)}{n}\right)$. In this section, we will use elements of $G$ as indices of generating isometries in $\mathcal{O}_{n}$. 
For $g \in G$, define unitaries $U(g) \in \mathcal{F}_{n} \subset \mathcal{O}_{n}$ as

$$
U(g)=\sum_{h \in G}\langle g, h\rangle S_{h} S_{h}^{*}
$$

Note that they give rise to a 'unitary representation' of $G$. Now define an endomorphism $\lambda \in \operatorname{End}\left(\mathcal{O}_{n}\right)$ by

$$
\lambda\left(S_{g}\right)=\frac{1}{\sqrt{n}} \sum_{h \in G}\langle g, h\rangle S_{h} U(g)^{*} .
$$

The endomorphism $\lambda$ does not leave $\mathcal{D}_{n}$ invariant (unless $n=1$ ), as we have

$$
\lambda\left(S_{g} S_{g}^{*}\right)=\frac{1}{n} \sum_{h, k \in G}\langle g, h-k\rangle S_{h} S_{k}^{*},
$$

so for example

$$
\lambda\left(S_{e} S_{e}^{*}\right)=\frac{1}{n} \sum_{h, k \in G} S_{h} S_{k}^{*} .
$$

The unitary associated with $\lambda$ is equal to

$$
v_{\lambda}=\frac{1}{\sqrt{n}} \sum_{g, h, l \in G}\langle g, h-l\rangle S_{h} S_{l} S_{l}^{*} S_{g}^{*} .
$$

For each $h \in G$ define an isometry

$$
\widetilde{S}_{h}=\frac{1}{\sqrt{n}} \sum_{a \in G}\langle h, a\rangle S_{a}
$$

and let $\beta \in \operatorname{Aut}\left(\mathcal{O}_{n}\right)$ be given by

$$
\beta\left(S_{h}\right)=\widetilde{S}_{h}, \quad h \in G .
$$

It is easy to see that $\beta$ is a Bogolyubov automorphism. Note that $\lambda^{\prime}:=\lambda \circ \beta$ is a permutation endomorphism. Indeed, for each $h \in G$ we have

$$
\begin{aligned}
\lambda^{\prime}\left(S_{h}\right) & =\lambda\left(\widetilde{S}_{h}\right)=\frac{1}{\sqrt{n}} \sum_{a \in G}\langle h, a\rangle \lambda\left(S_{a}\right)=\frac{1}{n} \sum_{a, b, c \in G}\langle h, a\rangle\langle b, a\rangle\langle-c, a\rangle S_{b} S_{c} S_{c}^{*} \\
& =\sum_{b \in G} S_{b} S_{h+b} S_{h+b}^{*} .
\end{aligned}
$$


Let us now compute $\lambda^{2}$ (recall it is a canonical endomorphism in the sense of Longo). For $g \in G$ we have

$$
\begin{aligned}
\lambda(U(g)) & =\sum_{h \in G}\langle g, h\rangle \frac{1}{n} \sum_{k, l \in G}\langle h, k-l\rangle S_{k} S_{l}^{*}=\frac{1}{n} \sum_{h, k, l \in G}\langle h, g+k-l\rangle S_{k} S_{l}^{*} \\
& =\sum_{k \in G} S_{k} S_{g+k}{ }^{*},
\end{aligned}
$$

and further

$$
\begin{aligned}
\lambda^{2}\left(S_{g}\right) & =\frac{1}{\sqrt{n}} \sum_{h \in G}\langle g, h\rangle \lambda\left(S_{h}\right) \lambda(U(g))^{*} \\
& =\frac{1}{n} \sum_{h \in G}\langle g, h\rangle \sum_{l \in G}\langle h, l\rangle S_{l} U(h)^{*} \sum_{k \in G} S_{g+k} S_{k}^{*} \\
& =\frac{1}{n} \sum_{h, l, k \in G}\langle g+l, h\rangle S_{l} \sum_{a \in G}\langle h,-a\rangle S_{a} S_{a}^{*} S_{g+k} S_{k}^{*} \\
& =\frac{1}{n} \sum_{h, l, k \in G}\langle g+l, h\rangle S_{l}\langle h,-g-k\rangle S_{g+k} S_{k}^{*} \\
& =\frac{1}{n} \sum_{h, l, k \in G}\langle l-k, h\rangle S_{l} S_{g+k} S_{k}^{*} \\
& =\sum_{k \in G} S_{k} S_{g+k} S_{k}^{*} .
\end{aligned}
$$

We can see from the formula above that $\lambda^{2}$ is a permutation endomorphism; its associated unitary is equal to

$$
v_{\lambda^{2}}=\sum_{g, h \in G} S_{g} S_{h+g} S_{g}^{*} S_{h}^{*} .
$$

Below, we take a closer look at the simplest nontrivial case, $G=\mathbb{Z}_{2}$.

Example 6.2. Let $G=\mathbb{Z}_{2}=\{0,1\}$ be equipped with the natural duality bracket; $\langle 1,1\rangle=-1$ and all other brackets take value 1 . The Izumi endomorphism discussed above is then given by

$$
\begin{aligned}
& \lambda\left(S_{0}\right)=\frac{1}{\sqrt{2}}\left(S_{0}+S_{1}\right), \\
& \lambda\left(S_{1}\right)=\frac{1}{\sqrt{2}}\left(S_{0} S_{0} S_{0}^{*}+S_{1} S_{1} S_{1}^{*}-S_{1} S_{0} S_{0}^{*}-S_{0} S_{1} S_{1}^{*}\right) .
\end{aligned}
$$


The unitary associated with $\lambda$ is given by

$$
\begin{aligned}
v_{\lambda} & =\frac{1}{\sqrt{2}}\left(S_{00,00}+S_{11,11}+S_{01,10}+S_{00,01}+S_{10,00}+S_{11,10}-S_{10,01}-S_{01,11}\right) \\
& =\frac{1}{\sqrt{2}}\left(S_{00,01}+S_{11,11}-S_{01,11}-S_{10,01}+S_{0,0}+S_{1,0}\right) \\
& =\frac{1}{\sqrt{2}}\left(1+S_{0,1}+S_{1,0}-2 S_{01,11}-2 S_{10,01}\right),
\end{aligned}
$$

where we write $S_{i, j}:=S_{i} S_{j}^{*}, S_{i j, k l}:=S_{i} S_{j} S_{k}^{*} S_{l}^{*}$ for all $i, j, k, l \in\{0,1\}$.

The permutation endomorphism $\lambda^{\prime}$ considered above is induced by the unitary

$$
v_{\lambda^{\prime}}=S_{00,00}+S_{01,11}+S_{11,01}+S_{10,10}
$$

and equals $\rho_{24}$ of [24]. Endomorphism $\lambda^{2}$ is associated to the unitary

$$
v_{\lambda^{2}}=S_{00,00}+S_{01,01}+S_{11,10}+S_{10,11}
$$

and equals $\rho_{243}$ of 24 .

It turns out that endomorphism $\lambda$ does not leave any standard MASA of $\mathcal{O}_{2}$ invariant. Indeed, let $z \in \mathcal{U}\left(\mathcal{F}_{2}^{1}\right)$. Then $\lambda$ preserves MASA $\lambda_{z^{*}}\left(\mathcal{D}_{2}\right)$ if and only if the endomorphism associated with $X=\lambda_{z}\left(v_{\lambda}\right)$ preserves $\mathcal{D}_{2}$ (Proposition 3.2). For this, it is necessary that $X \mathcal{D}_{2}^{1} X^{*} \subset \mathcal{D}_{2}^{2}$. So let

$$
z=a S_{0,0}+b S_{0,1}-\bar{b} S_{1,0}+\bar{a} S_{1,1}
$$

for some $a, b \in \mathbb{C}$ with $|a|^{2}+|b|^{2}=1$ (clearly, it suffices to consider unitaries $z$ with determinant 1 ). Then we have $X=\frac{1}{\sqrt{2}} z Y z^{*}$, where

$$
Y=1+S_{0,1}+S_{1,0}-2 S_{0} z S_{1} S_{1}^{*} z^{*} S_{1}^{*}-2 S_{1} z S_{0} S_{0}^{*} z^{*} S_{1}^{*}
$$

We verify that $X P_{0} X^{*}$ does not belong to $\mathcal{D}_{2}^{2}$ for any choice of parameters $a, b$. Indeed, since $z$ is in the commutant of $\varphi\left(\mathcal{F}_{2}^{1}\right)$, for $X P_{0} X^{*}$ to be in $\mathcal{D}_{2}^{2}$ it is necessary that $Y\left(z^{*} P_{0} z\right) Y^{*}$ is in $\mathcal{F}_{2}^{1} \varphi\left(\mathcal{D}_{2}^{1}\right)$. For computational convenience, we write $Y$ and $z^{*} P_{0} z$ as 4 -by-4 matrices in block form. Then

$$
Y=\left[\begin{array}{ll}
1 & Y_{1} \\
1 & Y_{2}
\end{array}\right] \quad \text { and } \quad z^{*} P_{0} z=\left[\begin{array}{cc}
c 1 & d 1 \\
\bar{d} 1 & (1-c) 1
\end{array}\right]
$$

where $c=|a|^{2}, d=\bar{a} b$ and $Y_{1}, Y_{2}$ are self-adjoint 2-by-2 matrices such that

$$
Y_{1}=\left[\begin{array}{cc}
2 a \bar{a}-1 & -2 a b \\
-2 \overline{a b} & 1-2 a \bar{a}
\end{array}\right], \quad Y_{2}=\left[\begin{array}{cc}
1-2 a \bar{a} & 2 a b \\
2 \overline{a b} & 2 a \bar{a}-1
\end{array}\right] .
$$


Thus $Y\left(z^{*} P_{0} z\right) Y^{*}$ equals

$$
\left[\begin{array}{cc}
c 1+(d+\bar{d}) Y_{1}+(1-c) Y_{1}^{2} & c 1+d Y_{2}+\bar{d} Y_{1}+(1-c) Y_{1} Y_{2} \\
c 1+\bar{d} Y_{2}+d Y_{1}+(1-c) Y_{2} Y_{1} & c 1+(d+\bar{d}) Y_{2}+(1-c) Y_{2}^{2}
\end{array}\right] .
$$

This is an element of $\mathcal{F}_{2}^{1} \varphi\left(\mathcal{D}_{2}^{1}\right)$ if and only if all its four blocks are diagonal 2-by-2 matrices. An elementary calculation shows that this happens if and only if either $a=0$ or $b=0$. But for these two choices of the parameters one can easily see that $X P_{0} X^{*}$ does not belong to $\mathcal{D}_{2}^{2}$.

\section{References}

[1] J.-C. Birget, The groups of Richard Thompson and complexity, Internat. J. Algebra Comput. 14 (2004), 569-626.

[2] O. Bratteli and D. E. Evans, Derivations tangential to compact groups: the non-abelian case, Proc. London Math. Soc. (3) 52 (1986), 369-384.

[3] R. Conti and F. Fidaleo, Braided endomorphisms of Cuntz algebras, Math. Scand. 87 (2000), 93-114.

[4] R. Conti, J. Kimberley and W. Szymański, More localized automorphisms of the Cuntz algebras, arXiv:0808.2843, to appear in Proc. Edinburgh Math. Soc.

[5] R. Conti and C. Pinzari, Remarks on the index of endomorphisms of Cuntz algebras, J. Funct. Anal. 142 (1996), 369-405.

[6] R. Conti, M. Rørdam and W. Szymański, Endomorphisms of $\mathcal{O}_{n}$ which preserve the canonical UHF-subalgebra, arXiv:0910.1304.

[7] R. Conti and W. Szymański, Computing the Jones index of quadratic permutation endomorphisms of $\mathcal{O}_{2}$, J. Math. Phys. 50 (2009), 012705.

[8] R. Conti and W. Szymański, Labeled trees and localized automorphisms of the Cuntz algebras, arXiv:0805.4654, to appear in Trans. Amer. Math. Soc.

[9] J. Cuntz, Simple $C^{*}$-algebras generated by isometries, Commun. Math. Phys. 57 (1977), 173-185. 
[10] J. Cuntz, Automorphisms of certain simple $C^{*}$-algebras, in Quantum fields-algebras-processes, ed. L. Streit, Springer 1980.

[11] J. Cuntz, Regular actions of Hopf algebras on the $C^{*}$-algebra generated by a Hilbert space, in Operator algebras, mathematical physics and low dimensional topology (R. Herman, B. Tanbay eds.), Res. Notes in Math. 5 (1993), 87-100.

[12] K. J. Dykema and D. Shlyakhtenko, Exactness of Cuntz-Pimsner $C^{*}$ algebras, Proc. Edinburgh Math. Soc. (2) 44 (2001), 425-444.

[13] J.-S. Huang, Lectures on representation theory, World Scientific Publ., River Edge, 1999.

[14] M. Izumi, Subalgebras of infinite $C^{*}$-algebras with finite Watatani indices. I. Cuntz algebras, Commun. Math. Phys. 155 (1993), 157-182.

[15] K. Kawamura, Polynomial endomorphisms of the Cuntz algebras arising from permutations. I. General theory, Lett. Math. Phys. 71 (2005), 149158.

[16] K. Kawamura, Branching laws for polynomial endomorphisms of Cuntz algebras arising from permutations, Lett. Math. Phys. 77 (2006), 111126.

[17] B. P. Kitchens, Symbolic dynamics. One-sided, two-sided and countable state Markov shifts, Springer-Verlag, Berlin, 1998.

[18] R. Longo, Index of subfactors and statistics of quantum fields. II. Correspondences, braid group statistics and Jones polynomial, Commun. Math. Phys. 130 (1990), 285-309.

[19] R. Longo, A duality for Hopf algebras and for subfactors. I, Commun. Math. Phys. 159 (1994), 133-150.

[20] V. Nekrashevych, Cuntz-Pimsner algebras of group actions, J. Operator Theory 52 (2004), 223-249.

[21] G. Price, Extremal traces on some group-invariant $C^{*}$-algebras, J. Funct. Anal. 49 (1982), 145-151. 
[22] A. Skalski, On automorphisms of $C^{*}$-algebras whose Voiculescu entropy is genuinely noncommutative, arXiv:0911.3951.

[23] A. Skalski, Noncommutative topological entropy of endomorphisms of Cuntz algebras II, preprint, November 2009.

[24] A. Skalski and J. Zacharias, Noncommutative topological entropy of endomorphisms of Cuntz algebras, Lett. Math. Phys. 86 (2008), 115-134.

[25] W. Szymański, On localized automorphisms of the Cuntz algebras which preserve the diagonal subalgebra, in 'New Development of Operator Algebras', R.I.M.S. Kôkyûroku 1587 (2008), 109-115.

[26] Y. Watatani, Index for $C^{*}$-subalgebras, Mem. Amer. Math. Soc. 83 (1990), no. 424.

Jeong Hee Hong

Department of Applied Mathematics

Korea Maritime University

Busan 606-791, South Korea

E-mail: hongjh@hhu.ac.kr

Adam Skalski

Department of Mathematics and Statistics

Lancaster University

Lancaster, LA1 4YF, United Kingdom 1

E-mail: a.skalski@lancaster.ac.uk

Wojciech Szymański

Department of Mathematics and Computer Science

The University of Southern Denmark

Campusvej 55, DK-5230 Odense M, Denmark

E-mail: szymanski@imada.sdu.dk

\footnotetext{
${ }^{1}$ Permanent address: Faculty of Mathematics and Computer Science, University of Lódź, ul. Banacha 22, 90-238 Lódź, Poland
} 